\title{
Biodiversidad de algas rojas marinas (Rhodophyta) en Tabasco, México
}

\section{Biodiversity of marine red algae (Rhodophyta) in Tabasco, Mexico}

\author{
Nataly Quiroz-González,3 [D, Daniel León-Álvarez [D, Ma. Guadalupe Rivas-Acuña² (D)
}

1 Universidad Nacional Autónoma de México, Facultad de Ciencias, Herbario, Laboratorio de Ficología y Sección de Algas, 04510 Cd. Mx., México.

2 Universidad Juárez Autónoma de Tabasco, División Académica de Ciencias Biológicas, 0.5 km carretera Cárdenas, 86000 Villahermosa, Tabasco, México.

3 Autor para la correspondencia: natalyquirozgonzalez@gmail.com

Citar como:

Quiroz-González, N., D. León-Álvarez y M. G. Rivas-Acuña. 2018. Biodiversidad de algas rojas marinas (Rhodophyta) en Tabasco, México. Acta Botanica Mexicana 123: 103-120. DOI: http://dx.doi. org/10.21829/abml23.2018.1253

Recibido: 2 de junio de 2017.

Revisado: 24 de julio de 2017

Aceptado: 31 de octubre de 2017.

Primero en línea: 2 de marzo de 2018

Publicado: 1 de abril de 2018.

DOI:

http://dx.doi.org/10.21829/abml23.2018 1253

\section{Resumen:}

Antecedentes y Objetivos: El conocimiento de la ficoflora en el estado de Tabasco es escaso. Hasta el momento se tienen 39 registros de Rhodophyta, 7 de Ochrophyta y 22 de Chlorophyta. Además, a nivel nacional es el estado costero con el menor número de publicaciones sobre el tema. Se desarrolló el presente trabajo para contribuir al conocimiento de la flora algal del estado, particularmente al de Rhodophyta.

Métodos: Se revisó material procedente de los herbarios de la Facultad de Ciencias (FCME) de la Universidad Nacional Autónoma de México (UNAM) y de la Universidad Juárez Autónoma de Tabasco (UJAT). Se llevaron a cabo dos periodos de muestreo en temporada de lluvias y secas del año 2012, en siete localidades del litoral tabasqueño. El material recolectado fue preservado en formol a $4 \%$. Se realizaron observaciones de talos completos y cortes con microscopios estereoscópico y óptico. La identificación se llevó a cabo con literatura especializada.

Resultados clave: Se reportan 12 nuevos registros de especies de algas rojas marinas para la costa de Tabasco pertenecientes a 11 géneros, 9 familias y 8 órdenes. En total para Tabasco se presentan 52 especies, 32 géneros, 17 familias y 10 órdenes. Los órdenes con mayor riqueza específica fueron Ceramiales y Gracilariales con 24 y 9 especies, respectivamente. Las familias con mayor número de especies fueron Rhodomelaceae y Gracilariaceae con 14 y 8 ocho cada una. El género más diverso fue Gracilaria, con ocho especies.

Conclusiones: El número de algas marinas rojas reportadas en Tabasco asciende de 39 a 52 especies, lo que representa $20 \%$ de las especies de Rhodophyta registradas para el Golfo de México.

Palabras clave: Golfo de México, macroalgas, riqueza específica, rodofita.

\section{ABSTRACT:}

Background and Aims: The knowledge of the phycoflora in the state of Tabasco is scarce. There are 39 records of Rhodophyta, 7 of Ochrophyta and 22 of Chlorophyta. In addition, at national level it is the coastal state with the lowest number of publications on the subject. The present work was developed to contribute to the knowledge of the algal flora of the state, particularly that of Rhodophyta.

Methods: Material from the herbaria of the Faculty of Sciences (FCME) of the Universidad Nacional Autónoma de Mexico (UNAM) and the Universidad Juárez Autónoma de Tabasco (UJAT) was studied. Two sampling periods were carried out in dry and rainy season of 2012, in seven localities of the coast of Tabasco. The collected material was preserved in formaldehyde at $4 \%$. Sections were performed with stereoscopic and optical microscopes. Identification was carried out with specialized literature.

Key results: Twelve new records of marine red algae species are reported from the coast of Tabasco, belonging to 11 genera, 9 families and 8 orders. In total, for Tabasco there are 52 species, 32 genera, 17 families and 10 orders. The orders with the highest species richness are Ceramiales and Gracilariales with 24 and 9 species, respectively. The families with the highest number of species are Rhodomelaceae and Gracilariaceae with 14 and 8 each one. The most diverse genus was Gracilaria, with eight species.

Conclusions: The number of red seaweeds recorded in Tabasco raises from 39 to 52 reports, representing $20 \%$ of the Rhodophyta species recorded for the Gulf of Mexico.

Key words: Gulf of Mexico, macroalgae, rhodophytes, specific richness. 


\section{INTRODUCCIÓN}

Los estudios de diversos autores, para conocer la ficoflora del Golfo de México (González-González et al., 1996; Littler y Littler, 2000; Ortega et al., 2001; Garduño-Solórzano et al., 2005), han sido realizados principalmente en los estados de Tamaulipas, Veracruz, Campeche y Yucatán. El conocimiento que se tiene de la flora ficológica de Tabasco es escaso, lo que se refleja en el bajo número de reportes de especies para el estado. Como lo señalan Quiroz-González et al. (2017) los estudios de mayor importancia realizados sobre las algas marinas en Tabasco son los de Orozco-Vega y Dreckmann (1995), Ramírez (1996), Dreckmann y De Lara-Issasi (2000), y Sentíes y Dreckmann (2013), quienes en total dan a conocer 71 taxa de macroalgas marinas para el litoral tabasqueño, de las cuales 39 corresponden a las algas rojas.

El objetivo de este estudio es contribuir al conocimiento ficoflorístico de algas marinas Rhodophyta del estado de Tabasco. Se presenta un listado florístico y se incluyen las descripciones e ilustraciones de los nuevos registros para este estado.

\section{Materiales y Métodos}

\section{Zona de estudio}

El estado de Tabasco está ubicado en el sureste de la República Mexicana entre las coordenadas $17^{\circ} 15^{\prime} 00^{\prime \prime}$ y $18^{\circ} 39^{\prime} 07^{\prime \prime}$ de latitud Norte y $90^{\circ} 50^{\prime} 23^{\prime \prime}$ y $94^{\circ} 07^{\prime} 49^{\prime \prime}$ de lon- gitud Oeste. Limita al norte con el Golfo de México, al noroeste con Campeche, al sureste con Guatemala, al sur con Chiapas y al oeste con Veracruz. El clima es de tipo cálidohúmedo, con una media anual de $26^{\circ} \mathrm{C}$ (West et al., 1985).

$\mathrm{Su}$ zona costera posee una ligera inclinación hacia el Golfo de México; se caracteriza por ser una planicie sedimentaria originada de la fuente interior de los ríos y la consecuente sedimentación se hace a través de humedales, estuarios, llanuras de inundación, manglares y/o pantanos marinos. Las playas bajas arenosas están constituidas principalmente por arcillas, limos, arenas y materia orgánica; también se presentan sedimentos no consolidados, los cuales están sujetos a la acción constante del oleaje (West et al., 1985). Dichas características no favorecen el desarrollo de muchas especies de algas bentónicas.

El litoral del estado de Tabasco presenta $191 \mathrm{~km}$ de longitud (Sánchez y Barba, 2005). Las localidades de estudio pertenecen a los municipios de Cárdenas, Centla y Paraíso (Cuadro 1, Fig. 1). Cárdenas presenta cerca de 67 $\mathrm{km}$ de costa y posee varias lagunas costeras de extensión considerable, tales como las lagunas El Carmen, Pajonal, Machona y Redonda. No se encuentran desembocaduras de ríos en este municipio. Centla tiene cerca de $75 \mathrm{~km}$ de costa en donde desembocan los ríos González, Grijalva, San Pedro y San Pablo. Paraíso posee alrededor de $38 \mathrm{~km}$ de costa y en su territorio se encuentra la Laguna de Mecoacán, la cual es de gran importancia económica. En su costa desembocan los ríos Seco, González y Verde.

Cuadro 1: Localidades de muestreo en el estado de Tabasco, México.

\begin{tabular}{lll}
\hline Municipio & Localidad & Georreferencia \\
\hline Cárdenas & Puerto Andrés Sánchez Magallanes & $18^{\circ} 17^{\prime} 42.91^{\prime \prime N} / 93^{\circ} 50^{\prime} 49.33^{\prime \prime O}$ \\
& Playa las Brisas & $18^{\circ} 17^{\prime} 26.65^{\prime \prime N} / 93^{\circ} 52^{\prime} 20.38^{\prime \prime O}$ \\
Centla & Playa Pico de Oro & $18^{\circ} 27^{\prime} 30.86^{\prime \prime N} / 92^{\circ} 51^{\prime} 17.57^{\prime \prime O}$ \\
& Playa Miramar & $18^{\circ} 29^{\prime} 00.34^{\prime \prime} \mathrm{N} / 92^{\circ} 47^{\prime} 44.65^{\prime \prime O}$ \\
Paraíso & Playa Paraíso & $18^{\circ} 25^{\prime} 47.93^{\prime \prime} \mathrm{N} / 93^{\circ} 12^{\prime} 4.60^{\prime \prime O}$ \\
& Puerto de dos Bocas & $18^{\circ} 26^{\prime} 39.77^{\prime \prime} \mathrm{N} / 93^{\circ} 07^{\prime} 12.29^{\prime \prime O}$ \\
& Poblado Chiltepec & $18^{\circ} 25^{\prime} 43.29^{\prime \prime N} / 93^{\circ} 05^{\prime} 00.88^{\prime \prime O}$ \\
\hline
\end{tabular}



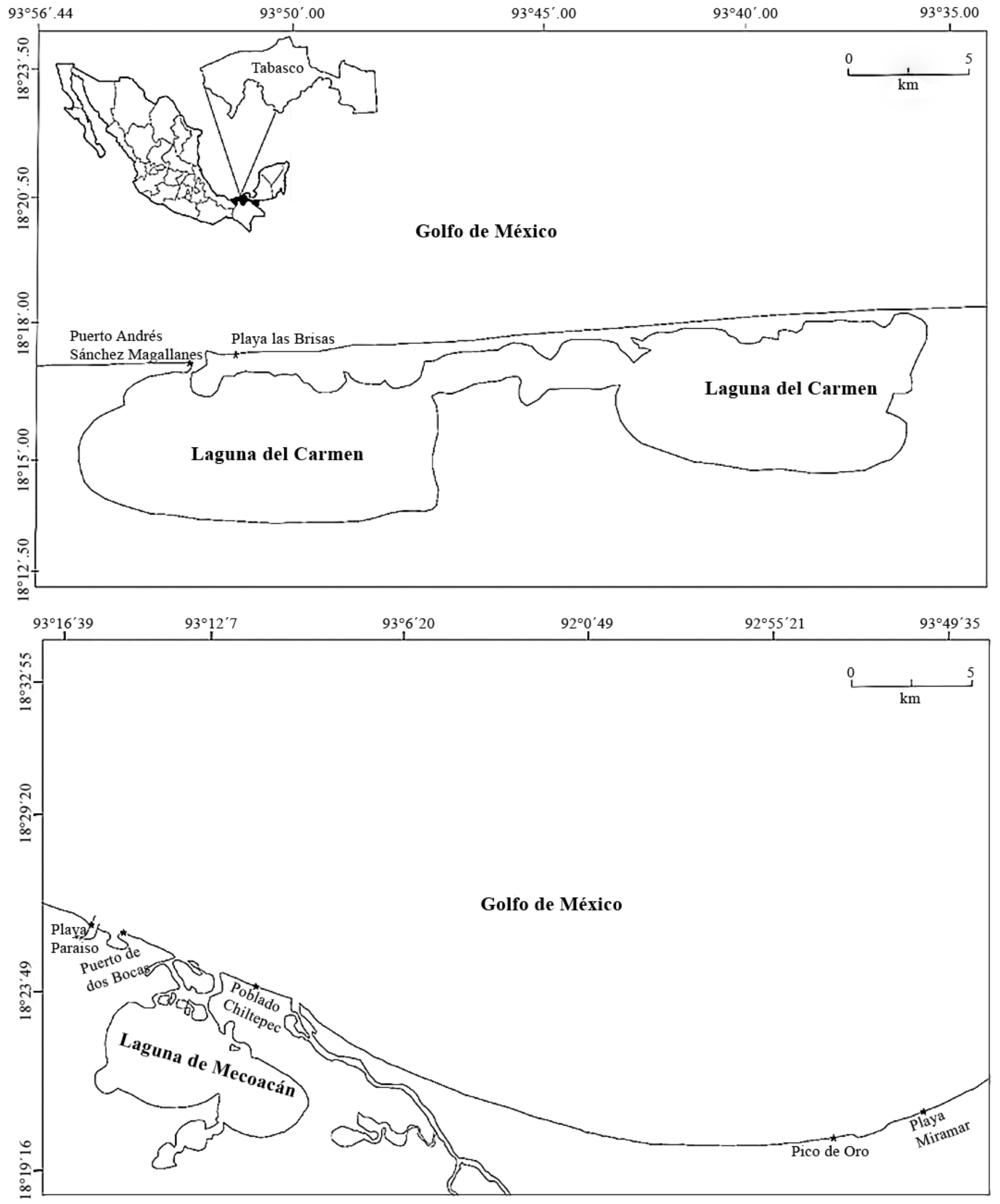

Figura 1: Ubicación del área de estudio y de las localidades de muestreo: Puerto Andrés Sánchez Magallanes y Playa las Brisas (municipio Cárdenas), Playa Pico de Oro y Playa Miramar (municipio Centla) y Playa Paraíso, Puertos de dos Bocas y Poblado Chiltepec (municipio Paraíso). 


\section{Trabajo de herbario y de campo}

Se realizó una revisión del material ficológico procedente de Tabasco en las colecciones de los herbarios de la Facultad de Ciencias (FCME) de la Universidad Nacional Autónoma de México y en el herbario de la Universidad Juárez Autónoma de Tabasco (UJAT). Con los datos recabados se determinaron los sitios y la temporada de muestreo para el trabajo de campo.

Paralelamente se efectuaron tres exploraciones ficológicas en la zona de estudio durante la época de secas (en mayo de 2012) y la época de lluvias (en octubre de 2012). Los organismos se recolectaron manualmente con espátula y navaja de campo en la zona intermareal y en las escolleras. Para cada ejemplar se tomaron las georreferencias con un GPS (GARMIN 60 CSx, Kansas, EUA) y se anotaron datos ecológicos como epifitismo y tipo de sustrato. Las muestras se trasladaron al herbario de la Universidad Juárez Autónoma de Tabasco (UJAT) y se fijaron con formol a 4\% en agua de mar (Lot y Chiang, 1986).

Se llevó a cabo una búsqueda bibliográfica para conocer los registros de algas rojas previamente señalados para el litoral tabasqueño.

\section{Procesamiento y descripción de muestras}

Los ejemplares se observaron con estereoscopio (Olympus SZ2-IL-ST, Tokio, Japón y ZEIGEN ZEZEZ2100, Cd. Mx., México). Se realizaron preparaciones semipermanentes y en fresco, se llevaron a cabo cortes histológicos o de secciones del talo en forma transversal. Los cortes se realizaron manualmente con una navaja de disección, los montajes semipermanentes se fijaron con gelatina glicerinada a $75 \%$, teñida con cristal violeta (Castellaro et al., 2007). Para la observación y toma de medidas celulares se empleó un microscopio compuesto (Olympus modelo CX31RTSF, Tokio, Japón). Se realizaron las descripciones e ilustraciones de los nuevos registros.

\section{Identificación taxonómica}

Se determinó el material ficológico con el uso de las siguientes obras: Taylor (1960), Littler et al. (1989), Littler y Littler (2000), De la Garza (2003) y Peruzzi et al. (2009).
Para la actualización nomenclatural se consultó AlgaeBase (Guiry y Guiry, 2017) y para el arreglo filogenético de clase y orden se utilizó a Mitsunobu et al. (2017); las familias, géneros y especies tienen un arreglo alfabético. Además, se compararon caracteres taxonómicos con los citados por Ramírez (1995), Mendoza-González y Mateo-Cid (1996), Guimarães et al. (2004), Castro-Nunes (2007), Fernández (2008), Ardito y García (2009), Morón y Ardito (2010), Solé y Pardo (2010), Solé y Suárez (2010) y Won et al. (2011).

Se integraron los números de cada preparación y las especies identificadas en la base de datos del sistema de información de la sección de algas del herbario FCME de la Universidad Nacional Autónoma de México y del herbario de la Universidad Juárez Autónoma de Tabasco (UJAT). Las muestras fueron depositadas en este último.

\section{Resultados}

Con este estudio, para Tabasco se registran en total 52 especies de rodofitas marinas, pertenecientes a 32 géneros, 17 familias y 10 órdenes (Cuadro 2). Se presentan además dos ejemplares que solo fueron identificados a nivel de género. De las especies listadas en este trabajo, 12 son nuevos registros para Tabasco. Los órdenes con mayor riqueza de especies son Ceramiales y Gracilariales con 24 y 9 especies, respectivamente. A su vez las familias con mayor número de especies fueron Rhodomelaceae y Gracilariaceae con 14 y 8 cada una, mientras que el género más diverso fue Gracilaria con ocho. Con este trabajo el número de taxa de Rhodophyceae para Tabasco ascendió de 39 a 52 , lo que representa $20 \%$ de las especies reportadas para el Golfo de México (Cuadro 2).

El municipio con mayor número de especies fue Cárdenas con 24 seguido de Paraíso con 13. Cinco especies se encontraron en ambos municipios. La localidad con el mayor número de especies registradas (22) fue el Puerto Andrés Sánchez Magallanes (Cuadro 2).

Las algas se encontraron sobre distintos sustratos: $59 \%$ de las especies sobre arena, limo y sustratos artificiales como escolleras, dragas, placas de cemento, basura (plásticos, cuerdas), costales y telas, $41 \%$ en otras algas, pastos marinos, troncos y conchas de animales. Únicamente Cera- 
Cuadro 2: Especies de Rhodophyta registradas para Tabasco. Localidades: Puerto Andrés Sánchez Magallanes (SM), Playa las Brisas (PB), Pico de Oro (PO), Playa Miramar (PM), Puerto de dos Bocas (PDB), Playa Paraíso Escollera Oeste (PPEO), Poblado Chiltepec (PCH). Especies reportadas en otras obras para Tabasco pero donde no se especifica la localidad: TAB. Sustratos: rocas (escolleras) (Ro), animales $(\mathrm{An})$, flotadoras (Fl), arena (Ar), algas (epífitas) (Al ), limo (Li), placas de cemento (Pc), plásticos (Pl), pastos marinos (Pm), costales (Co), cuerdas $(\mathrm{Cu})$, dragas (D), troncos (Tr), telas (Te). Acrónimos: Colección Algas (CA), Herbario de la Universidad Juárez Autónoma de Tabasco (UJAT), Golfo de México (GM), Herbario de la Facultad de Ciencias de la UNAM (FCME). ND: datos no determinados. *Nuevo registro para el estado de Tabasco. ${ }^{1}$ Orozco-Vega y Dreckmann (1995), ${ }^{2}$ Ramírez (1996), ${ }^{3}$ Dreckmann y De Lara-Isassi (2000), ${ }^{4}$ Ortega et al. (2001), ${ }^{5}$ Sentíes y Dreckmann (2013).

\begin{tabular}{|c|c|c|c|c|c|c|c|c|c|c|c|}
\hline \multirow[b]{3}{*}{ Taxa } & \multicolumn{8}{|c|}{ Localidades } & \multirow[b]{3}{*}{ Sustrato } & & \\
\hline & \multicolumn{2}{|c|}{ Cárdenas } & \multicolumn{2}{|c|}{ Centla } & \multicolumn{3}{|c|}{ Paraíso } & \multirow[t]{2}{*}{ TAB } & & \multicolumn{2}{|c|}{ Referencias de herbario } \\
\hline & SM & PB & $\mathrm{PO}$ & PM & PDB & PPEO & $\mathrm{PCH}$ & & & FCME & UJAT \\
\hline \multicolumn{12}{|l|}{ Bangiophyceae } \\
\hline \multicolumn{12}{|l|}{ Bangiales } \\
\hline \multicolumn{12}{|l|}{ Bangiaceae } \\
\hline Bangia fuscopurpurea (Dillwyn) Lyngbye* & - & - & - & - & - & $\mathrm{X}$ & - & - & $\mathrm{Al}$ & GM391 & - \\
\hline \multicolumn{12}{|l|}{ Compsopogonophyceae } \\
\hline \multicolumn{12}{|l|}{ Erythropeltidales } \\
\hline \multicolumn{12}{|l|}{ Erythrotrichiaceae } \\
\hline Erythrotrichia carnea (Dillwyn) J. Agardh ${ }^{2,5}$ & $\mathrm{X}$ & - & - & - & - & - & - & - & $\mathrm{Al}$ & GM391 & - \\
\hline Sahlingia subintegra (Rosenvinge) Kornmann* & $\mathrm{X}$ & - & - & - & - & - & - & - & $\mathrm{Al}$ & - & CA132 \\
\hline \multicolumn{12}{|l|}{ Florideophyceae } \\
\hline \multicolumn{12}{|l|}{ Corallinales } \\
\hline \multicolumn{12}{|l|}{ Corallinaceae } \\
\hline \multirow[t]{6}{*}{ Jania adhaerens J.V. Lamouroux* } & - & - & - & - & - & $\mathrm{X}$ & - & - & Ro & GM394 & CA3 \\
\hline & & & & & & & & & & GM396 & \\
\hline & & & & & & & & & & GM398 & \\
\hline & & & & & & & & & & GM399 & \\
\hline & & & & & & & & & & GM400 & \\
\hline & & & & & & & & & & GM403 & \\
\hline J. cubensis Montagne ex Kützing ${ }^{5}$ & - & - & - & - & - & - & - & $\mathrm{X}$ & ND & - & - \\
\hline Pneophyllum fragile Kützing ${ }^{5}$ & - & - & - & - & - & - & - & $\mathrm{X}$ & ND & - & - \\
\hline
\end{tabular}

\section{Bonnemaisoniales}

Bonnemaisoniaceae

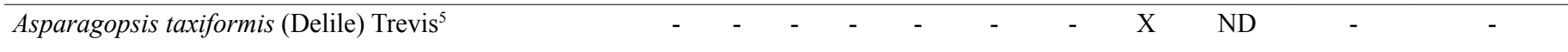

\section{Ceramiales}

\section{Ceramiaceae}

\begin{tabular}{|c|c|c|c|c|c|c|c|c|c|c|c|}
\hline $\begin{array}{l}\text { Aglaothamnion boergesenii (Aponte \& D.L. Ballantine) } \\
\text { L'Hardy-Halos \& Rueness }^{1,5}\end{array}$ & - & - & - & - & - & - & - & $\mathrm{X}$ & ND & - & - \\
\hline Callithamnion corymbosum (Smith) Lyngbye ${ }^{2,5}$ & - & - & - & - & - & - & - & $\mathrm{X}$ & $\mathrm{Al}$ & - & - \\
\hline & & & & & & & & & Ro, D & & CA132 \\
\hline & & & & & & & & & & & CA134 \\
\hline & & & & & & & & & & & CA164 \\
\hline & & & & & & & & & & & CA179 \\
\hline
\end{tabular}


Cuadro 2: Continuación.

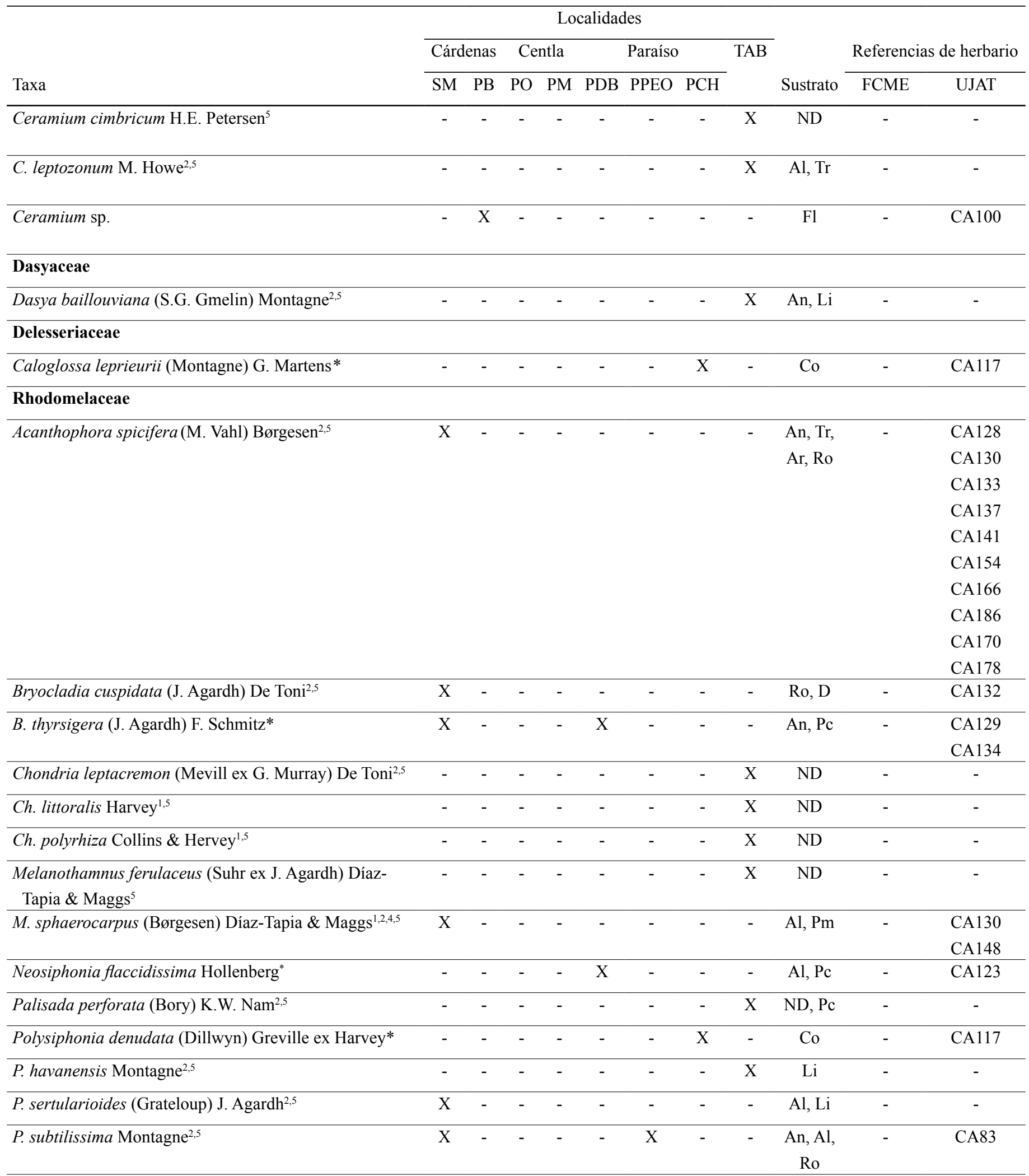


Cuadro 2: Continuación.

Localidades

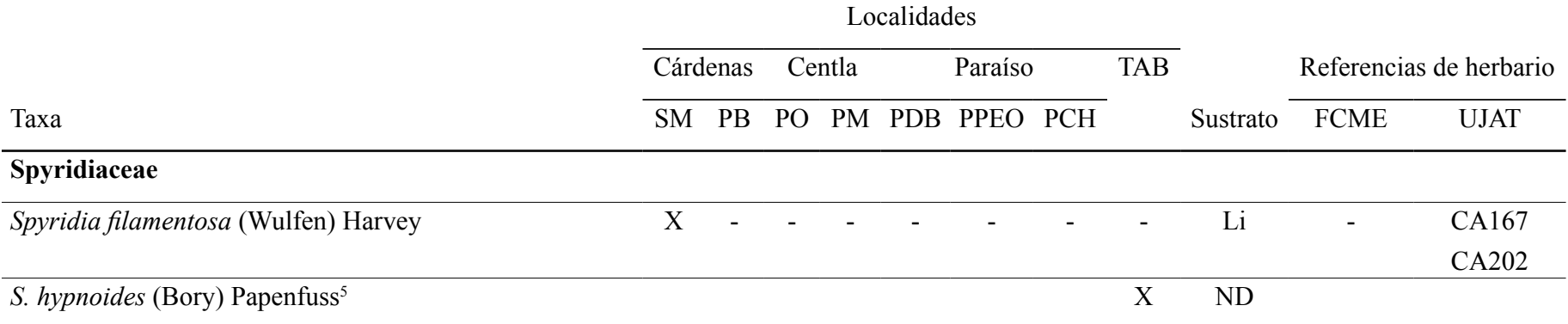

\section{Wrangeliaceae}

Tiffaniella gorgonea (Montagne) Doty \& Meñez

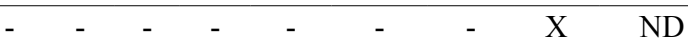

\section{Gelidiales}

Gelidiaceae

Gelidium pusillum (Stackhouse) Le Jolis ${ }^{2,5}$

X $\quad-\quad \begin{array}{lllllllll} & - & - & - & - & - & - & \text { Ro, An }\end{array}$

\section{Gigartinales}

Cystocloniaceae

Hypnea cervicornis J. Agardh ${ }^{2}$

H. musciformis (Wulfen) J.V. Lamouroux ${ }^{2,5}$

H. spinella (C. Agardh) Kützing ${ }^{2,5}$

H. valentiae (Turner) Montagne*

\begin{tabular}{|c|c|c|c|c|c|c|c|c|c|c|}
\hline- & $\mathrm{X}$ & - & - & $X$ & - & - & - & $\mathrm{Cu}, \mathrm{D}$ & - & - \\
\hline $\mathrm{X}$ & - & - & - & - & - & - & - & $\mathrm{Al}$ & - & CA93 \\
\hline $\mathrm{X}$ & - & - & - & - & - & - & - & ND & - & - \\
\hline \multirow[t]{8}{*}{$X$} & - & - & - & $X$ & $X$ & - & - & $\mathrm{Al}, \mathrm{Pm}$, & GM391 & CA120 \\
\hline & & & & & & & & Ar, Ro, & GM394 & CA128 \\
\hline & & & & & & & & $\mathrm{Li}$ & GM402 & CA130 \\
\hline & & & & & & & & & & CA133 \\
\hline & & & & & & & & & & CA139 \\
\hline & & & & & & & & & & CA141 \\
\hline & & & & & & & & & & CA142 \\
\hline & & & & & & & & & & CA145 \\
\hline
\end{tabular}

CA2 12

Solieriaceae

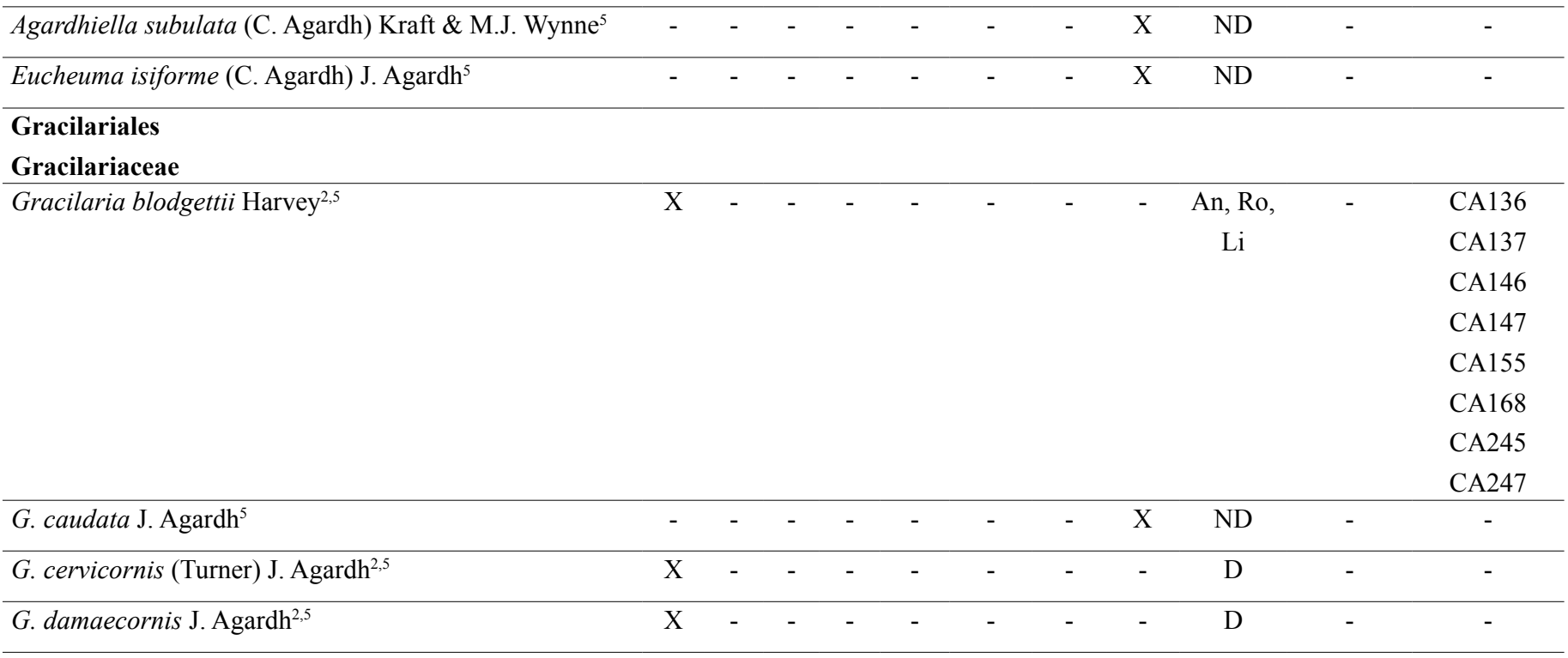




\begin{tabular}{|c|c|c|c|c|c|c|c|c|c|c|c|}
\hline \multirow[b]{3}{*}{ Taxa } & \multicolumn{8}{|c|}{ Localidades } & \multirow[b]{3}{*}{ Sustrato } & & \\
\hline & \multicolumn{2}{|c|}{ Cárdenas } & \multicolumn{2}{|c|}{ Centla } & \multicolumn{3}{|c|}{ Paraíso } & \multirow[t]{2}{*}{ TAB } & & \multicolumn{2}{|c|}{ Referencias de herbario } \\
\hline & $\mathrm{SM}$ & PB & $\mathrm{PO}$ & $\mathrm{PM}$ & PDB & PPEO & $\mathrm{PCH}$ & & & FCME & UJAT \\
\hline G. foliifera (Forsskål) Børgesen ${ }^{2,5}$ & $\mathrm{X}$ & - & - & - & - & - & - & - & ND & - & - \\
\hline G. pacifica I.A. Abbott ${ }^{1}$ & - & - & - & - & - & - & - & $\mathrm{X}$ & ND & - & - \\
\hline G. tikvahiae McLachlan* & - & - & - & - & $\mathrm{X}$ & - & - & - & Ar, Ro & - & CA212 \\
\hline G. venezuelensis W.R. Taylor* & $\mathrm{X}$ & - & - & - & - & - & - & - & Ro, Li & - & $\begin{array}{l}\text { CA136 } \\
\text { CA153 } \\
\text { CA156 } \\
\text { CA173 }\end{array}$ \\
\hline Gracilaria sp. & - & - & - & - & $\mathrm{X}$ & - & - & - & Ro & - & CA84 \\
\hline $\begin{array}{l}\text { Gracilariopsis tenuifrons (C.J. Bird \& E.C. Oliveira) } \\
\text { Fredericq \& Hommersand }{ }^{2}\end{array}$ & $\mathrm{X}$ & - & - & - & - & - & - & - & An & - & - \\
\hline $\begin{array}{l}\text { Halymeniales } \\
\text { Halymeniaceae }\end{array}$ & & & & & & & & & & & \\
\hline Grateloupia filicina (J.V. Lamouroux) C. Agardh ${ }^{2,5}$ & $\mathrm{X}$ & - & - & - & $\mathrm{X}$ & - & - & - & $\begin{array}{l}\mathrm{Al}, \mathrm{An}, \\
\mathrm{Ro}, \mathrm{Pl}, \\
\mathrm{Pc}, \mathrm{Cu}\end{array}$ & - & $\begin{array}{l}\text { CA129 } \\
\text { CA163 } \\
\text { CA174 } \\
\text { CA122 }\end{array}$ \\
\hline Halymenia floresii (Clemente) C. Agardh ${ }^{5}$ & - & - & - & - & - & - & - & $\mathrm{X}$ & ND & - & - \\
\hline $\begin{array}{l}\text { Rhodymeniales } \\
\text { Lomentariaceae }\end{array}$ & & & & & & & & & & & \\
\hline 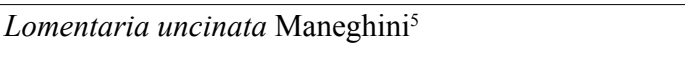 & - & - & - & - & - & - & - & $\mathrm{X}$ & ND & - & - \\
\hline Rhodymeniaceae & & & & & & & & & & & \\
\hline Chrysymenia enteromorpha Harvey ${ }^{5}$ & - & - & - & - & - & - & - & $\mathrm{X}$ & ND & - & - \\
\hline $\begin{array}{l}\text { Rhodymenia pseudopalmata (J.V. Lamouroux) P.C. } \\
\text { Silva* }\end{array}$ & $\mathrm{X}$ & - & - & - & - & - & - & - & $\mathrm{Pl}$ & $\begin{array}{c}\text { GM71 } \\
\text { GM394 } \\
\text { GM400 } \\
\text { GM403 } \\
\text { GM406 }\end{array}$ & $\begin{array}{c}\text { CA3 } \\
\text { CA179 }\end{array}$ \\
\hline
\end{tabular}

mium sp. se reporta como flotadora, aunque se desconoce si se trata de una especie flotadora como tal, o si es bentónica y se desprendió del sustrato (Cuadro 2). Las escolleras fueron el sustrato más frecuente seguidas de los de tipo biótico como las algas y conchas de ostión (Crassostrea virginica Gmelin).

Se encontraron 13 especies de algas epífitas de otras algas y/o de pastos marinos (Thalassia testudinum K.D. Koening): Erythrotrichia carnea (Dillwyn) J. Agardh, Bangia fuscopurpurea (Dillwyn) Lyngbye, Sahlingia subinte- gra (Rosenvinge) Kornmann, Callithamnion corymbosum (Smith) Lyngbye, Centroceras clavulatum (C. Agardh) Montagne, Ceramium leptozonum M. Howe, Hypnea valentiae (Turner) Montagne, Hypnea musciformis (Wulfen) J.V. Lamouroux, Grateloupia filicina (J.V. Lamouroux) C. Agardh, Neosiphonia flaccidissima (Hollenberg) M.S. Kim \& I.K. Lee, Melanothamnus sphaerocarpus (Børgesen) Díaz-Tapia \& Maggs, Polysiphonia subtilissima Montagne y P. sertularioides (Grateloup) J. Agardh. De éstas, Erythrotrichia car- 
nea y Sahlingia subintegra se encontraron únicamente como epífitas, es decir no se les observó en ningún otro sustrato.

\section{DESCRIPCIONES MORFOLÓGICAS}

\section{Rhodophyta}

\section{Bangiophyceae}

\section{Bangiales}

\section{Bangiaceae}

Bangia fuscopurpurea (Dillwyn) Lyngbye, Tent. Hydrophytol. Dan. 83. 1819. TIPO: INGLATERRA. Dunraven Castle, W. W. Young s.n. (no localizado). Fig. 2A.

Talo filamentoso, gregario, hasta $3 \mathrm{~mm}$ de largo, color púrpura, lubricoso, uniseriado, sin ramificar; pared celular gruesa, con constricciones, 20-32 $\mu \mathrm{m}$ de ancho; células de tamaño y forma irregular, 90-110 $\mu \mathrm{m}$ de diámetro, 82-115 $\mu \mathrm{m}$ de largo.

Hábitat: epífita de Chaetomorpha antennina Bory (Kützing).

Ejemplares examinados: MÉXICO. Tabasco, municipio Paraíso, Playa Paraíso, Escollera Oeste, 15.XIII.1989, D. León GM391 (FCME).

\section{Compsopogonophyceae}

\section{Erythropeltidales}

\section{Erythrotrichiaceae}

Sahlingia subintegra (Rosenvinge) Kornmann, $\mathrm{Br}$. Phycol. J. 24: 227, fig. 13. 1989. TIPO: DINAMARCA. Møllegrund, Skagerrak, off Hirshals. Sin colector. Figs. 2B, C.
Talos diminutos, 13-67 $\mu \mathrm{m}$ de diámetro, color rojo a rosa, monostromáticos; células dispuestas de forma radial para formar un disco, células centrales 2-3 $\mu \mathrm{m}$ de diámetro, 2-4 $\mu \mathrm{m}$ de largo, el resto de las células alargadas, 2-3.5 $\mu \mathrm{m}$ de diámetro, 2.5-7 $\mu \mathrm{m}$ de largo, células marginales con una bifurcación.

Hábitat: epífita estricta de Chaetomorpha antennina.

Ejemplares examinados: MÉXICO. Tabasco, municipio Cárdenas, Puerto Andrés Sánchez Magallanes, 27.X.2012, N. Quiroz CA132 (UJAT).

\section{Florideophyceae}

\section{Corallinales}

\section{Corallinaceae}

Jania adhaerens J.V. Lamouroux, Hist. Polyp. Corall. Flex: 270. 1816. TIPO: MAR MEDITERRÁNEO. Sin localidad. Figs. 2D, E.

Talo erecto, gregario, hasta $4 \mathrm{~cm}$ de alto, color rosa claro; ramificación dicotómica, hasta quinto orden, en un solo plano; ramas cilíndricas, 90-200 $\mu \mathrm{m}$ de diámetro que se adelgazan hacia los ápices; intergenículas fuertemente calcificadas, 0.3-0.8 mm de largo, se constriñen hacia las genículas; genículas sin calcificar, aparecen en la base de las ramas, en intervalos regulares entre estas; células elongadas, en una sola fila, $5-10 \mu \mathrm{m}$ de diámetro, 30-70 $\mu \mathrm{m}$ de largo; células medulares longitudinalmente dispuestas en filas paralelas, 62-74 $\mu \mathrm{m}$ de longitud; células corticales rectangulares a redondeadas, $7.5 \mu \mathrm{m}$ de diámetro; conceptáculos tetrasporangiales en forma de copa, 180-320 $\mu \mathrm{m}$ de diámetro, en la zona de surgimiento de las ramas, al final de una intergenícula; tetrasporangio oval a elíptico, 40-60 $\mu \mathrm{m}$ de diámetro, 80-110 $\mu \mathrm{m}$ de longitud.

Hábitat: rocas formadoras de escollera. 

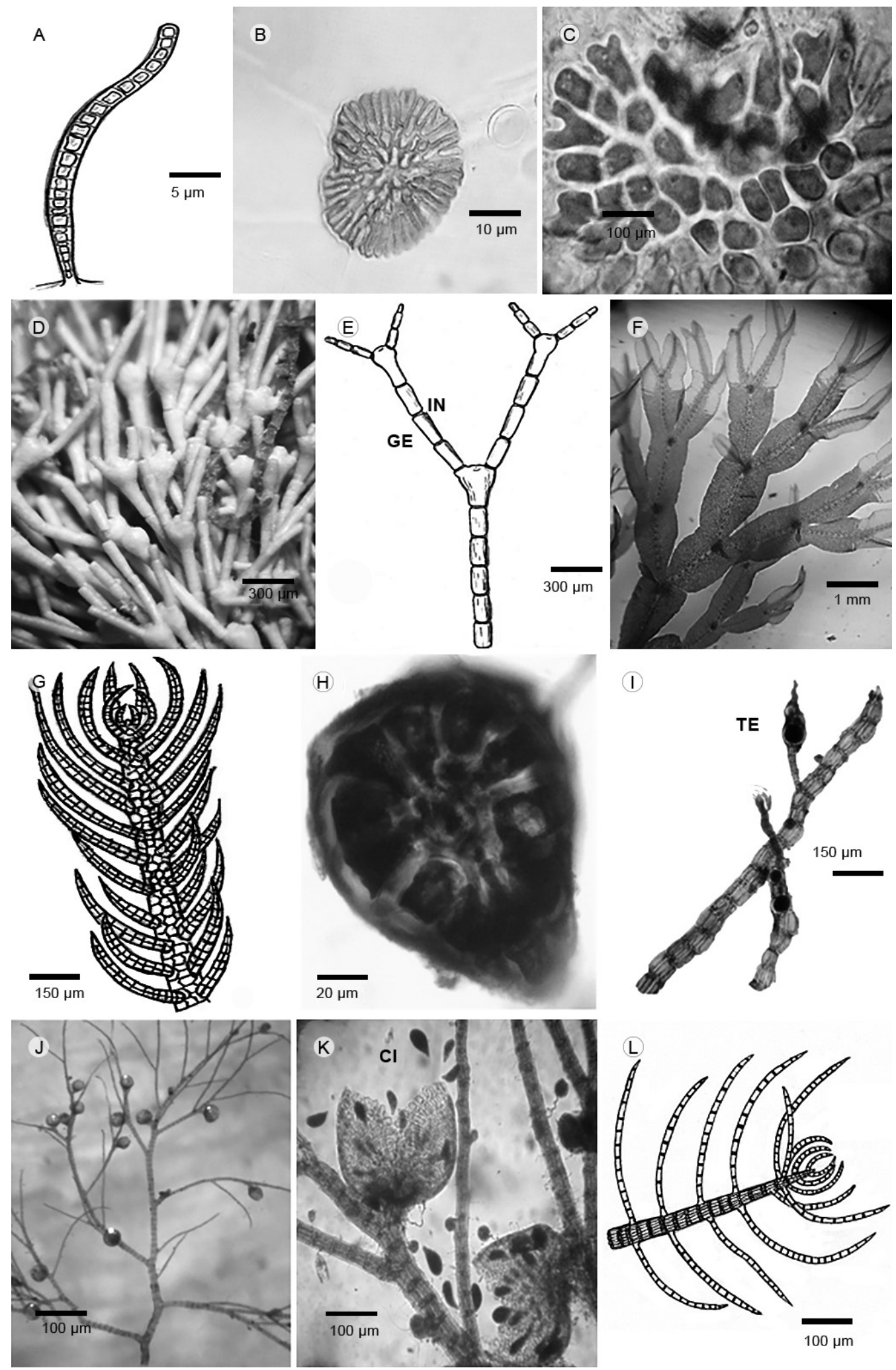

Figura 2: A. Bangia fuscopurpurea (Dillwyn) Lyngbye: aspecto del talo. B, C. Sahlingia subintegra (Rosenvinge) Kornmann: B. aspecto del talo; C. detalle células bifurcadas. D, E. Jania adhaerens V. Lamouroux: D. aspecto del talo; E. genículas (GE) e intergenículas (IN). F. Caloglossa leprieurii (Montagne) G. Martens: aspecto del talo. G, H. Bryocladia thyrsigera (J. Agardh) F. Schmitz: G. aspecto del talo; H. corte transversal. I-K. Polysiphonia denudata (Dillwyn) Greville ex Harvey: I. rama tetrasporangil (TE); J. talo con cistocarpos; K. cistocarpos con caposporas recién liberadas (CI). L. Spyridia filamentosa (J. Agardh) F. Schmitz: aspecto y corte transversal. 
Ejemplares examinados: MÉXICO. Tabasco, municipio Paraíso, Playa Paraíso Escollera Oeste, 15.VIII.1989, D. León GM394 (FCME), GM396 (FCME), GM398 (FCME), GM399 (FCME), GM400 (FCME), GM403 (FCME).

\section{Ceramiales}

\section{Delesseriaceae}

Caloglossa leprieurii (Montagne) G. Martens, Flora 52: 234, 237. 1869. TIPO: GUYANA FRANCESA. Sinnamary, al noreste de Cayena septentrional, Leprieur (no localizado). Fig. 2F.

Talo laminar erecto, hasta 4-5 cm de largo, 5-10 $\mathrm{mm}$ de ancho en las porciones basales, en la región media 350-500 $\mu \mathrm{m}$ de ancho, en los ápices 25-50 $\mu \mathrm{m}$ de ancho, constreñido hacia la base, color café-verdoso a violeta-verdoso; ramificación dicotómica; lámina monostromática; 1 nervadura central formada por 3 hileras de células, cada célula de forma rectangular, 5-7 $\mu \mathrm{m}$ de ancho, 12-54 $\mu \mathrm{m}$ de largo, el resto de las células son poliédricas dispuestas en hileras diagonales que van del margen del talo a la nervadura, 5-7 $\mu \mathrm{m}$ de ancho, 6-17 $\mu \mathrm{m}$ de largo; formación de rizoides en las constricciones, así como de segmentos del talo, también proliferan desde las nervaduras; no se observaron estructuras reproductivas.

Hábitat: sobre sustrato artificial (costales).

Ejemplares examinados: MÉXICO. Tabasco, municipio Paraíso, Poblado Chiltepec, 05.VII.2012, M. Ortiz CA117 (UJAT).

\section{Rhodomelaceae}

Bryocladia thyrsigera (J. Agardh) F. Schmitz, Fauna und Flora des Golfes von Neapel, Monographie 26: 169. 1901. TIPO: ITALIA. Sin localidad. Figs. 2G, H.
Talo hasta $10 \mathrm{~cm}$ de alto, color púrpura-rojizo a negro; ejes principales erectos, ecorticados, 90-140 $\mu \mathrm{m}$ de diámetro; ramificación alterna y radial en más de un plano, de hasta cuarto orden; ramillas pinnadas, curvadas hacia el eje principal, rígidas; 10 células pericentrales, 1 célula axial, células poliédricas o romboides, 18-26 $\mu \mathrm{m}$ de diámetro, 42-60 $\mu \mathrm{m}$ de largo; no se observaron estructuras reproductivas.

Hábitat: placas de cemento, conchas de moluscos.

Ejemplares examinados: MÉXICO. Tabasco, municipio Cárdenas, Puerto Andrés Sánchez Magallanes, 27.X.2012, N. Quiroz et al. CA129 (UJAT), CA134 (UJAT). Municipio Paraíso, Puerto de dos Bocas, 25.X.2012, N. Quiroz et al. CA123 (UJAT).

Neosiphonia flaccidissima (Hollenberg) M.S. Kim \& I.K. Lee, Phycol. Res. 47: 279. 1999. TIPO: ESTADOS UNIDOS DE AMÉRICA. California, Laguna Beach, Orange County, G. J. Hollenberg 2269 (no localizado).

Talo filamentoso, aspecto fino, color pardo-rojizo; ejes postrados 65-80 $\mu \mathrm{m}$ de diámetro, rizoides 10-20 $\mu \mathrm{m}$ de diámetro, 125-650 $\mu \mathrm{m}$ de largo; ejes erectos sin diferenciación de un eje principal, 40-55 $\mu \mathrm{m}$ de diámetro, hacia la zona apical el talo se adelgaza, 15-25 $\mu \mathrm{m}$ de diámetro; ramificaciones alternas, irregulares o pseudodicotómicas; ápice con presencia de una sola célula apical; 4 células pericentrales, 1 célula axial; tricoblastos en espiral, 1.2-2.4 $\mu \mathrm{m}$ de diámetro, 18-40 $\mu \mathrm{m}$ de largo.

Hábitat: epífita de Grateloupia filicina.

Ejemplares examinados: MÉXICO. Tabasco, municipio Paraíso, Puerto de dos Bocas, 25.X.2012, N. Quiroz et al. CA122 (UJAT).

Nota: esta especie es incierta ya que Guiry y Guiry (2017) la consideran como sinónimo de Neosiphonia sertularioides; sin embargo, Díaz-Tapia et al. (2017) seña- 
lan que el material tipo es del Mediterráneo y la especie del Atlántico no pertenece al género Melanothamnus ni a otros géneros relacionados. Por lo pronto retenemos esta especie con su nombre original.

Polysiphonia denudata (Dillwyn) Greville ex Harvey, English Flora 5(1): 332. 1833. TIPO: INGLATERRA. Southampton. Sin colector. Figs. 2I-K.

Talo filamentoso, hasta $8 \mathrm{~cm}$ de alto, color rojo vino; ejes principales erectos, 30-70 $\mu \mathrm{m}$ de diámetro hacia la zona media, 170-250 $\mu \mathrm{m}$ de diámetro en la región basal, con 3-4 hileras de células, tienden a adelgazar hacia los ápices; ramificación pseudodicotómica, ramas alternas hasta cuarto orden; 6 células pericentrales, 1 célula axial; ápices bifurcados, sumamente corticados, algunos monosifónicos, 10-25 $\mu \mathrm{m}$ de diámetro; tricoblastos ausentes; cistocarpos ovoides, 180-200 $\mu \mathrm{m}$ de diámetro.

Hábitat: sobre sustrato artificial (costales).

Ejemplares examinados: MÉXICO. Tabasco, municipio Paraíso, Poblado Chiltepec, 05.VII.2012, M. Ortiz CA117 (UJAT).

\section{Spyridiaceae}

Spyridia filamentosa (Wulfen) Harvey, English Flora 5(1): 336. 1833. TIPO: MAR ADRIÁTICO. Vienna, sin colector. Fig. 2L.

Talo filamentoso, de aspecto plumoso, $6.5 \mathrm{~cm}$ de alto, color rosa a pardo oscuro; ejes principales 1-1.5 mm de diámetro; ramificación alterno-dística, de hasta segundo orden; ramas secundarias dispuestas de forma dística o alterna en más de un plano, 66-100 $\mu \mathrm{m}$ de diámetro; ramillas insertas radialmente $20-46 \mu \mathrm{m}$ de diámetro, 0.5$1.5 \mathrm{~mm}$ de longitud, sin ramificar, segmentos ecorticados, 45-95 $\mu \mathrm{m}$ de longitud; ramillas con una sola célula apical; células superficiales en una sola hilera; no se observaron estructuras reproductivas.
Hábitat: limo.

Ejemplares examinados: MÉXICO. Tabasco, municipio Cárdenas, Puerto Andrés Sánchez Magallanes, 27.X.2012, A. Córdova CA167 (UJAT), CA202 (UJAT).

\section{Gigartinales}

\section{Cystocloniaceae}

Hypnea valentiae (Turner) Montagne, Hist. Nat. Iles Canaries 3: 161. 1841. TIPO: MAR ROJO. Lord Valentia s.n. (holotipo: BM (ex K)). Figs. 3A-C.

Talo cilíndrico, diámetro $0.5-1 \mathrm{~mm}$, hasta $2 \mathrm{~cm}$ de largo, color amarillo verdoso; ramificaciones irregulares de primer orden, en diferentes planos, enredadas, surgen de una base; ramas curvean hacia adentro con dirección a la base, ápices aguzados, ligeramente más aplanados que el resto del talo, ramas cortas parecen pequeños espolones o espinas, que en algunas zonas se conglomeran dando la apariencia de una estrella; 5 células pericentrales, 1 célula axial, 42-100 $\mu \mathrm{m}$ de diámetro, células medulares hialinas, ovaladas, poliédrica e irregular, de paredes gruesas; corteza 2 células de espesor, células corticales fuertemente pigmentadas de un tono color pardo amarillo, de forma ovalada a irregular, 7.5-12.5 $\mu \mathrm{m}$ de diámetro; cistocarpo $250 \mu \mathrm{m}$ de diámetro, 185 $\mu \mathrm{m}$ de largo.

Hábitat: epífita de pastos marinos. Gracilariales.

Ejemplares examinados: MÉXICO. Tabasco, municipio Cárdenas, Puerto Andrés Sánchez Magallanes, 27.X.2012, N. Quiroz et al. CA128 (UJAT), CA130 (UJAT), CA133 (UJAT), CA139 (UJAT), CA141 (UJAT), CA142 (UJAT), CA145 (UJAT); loc. cit., 02.IX.2012, O. Hernández CA120 (UJAT). Municipio Paraíso, Playa Paraíso Escollera Oeste, 15.VIII.1989, D. León GM391 (FCME), GM394 (FCME), GM402 (FCME); Puerto de dos Bocas, 26.V.2008, G. Hernández CA212 (UJAT). 


\section{Gracilariaceae}

Gracilaria tikvahiae McLachlan, Phycologia 18: 19. 1979. TIPO: CANADÁ. Barrachois Harbour, Colchester Co., Nova Scotia (holotipo: NRCC). Figs. 3D-E.

Talo liso, color café-rojizo, cilíndrico que se adelgaza hacia los ápices, 7.1-11.5 cm de largo, 1-2.5 mm de ancho; ramificación pseudodicotómica hacia la base, se torna irregular y radial en el resto del talo; ápices dicotómicos o tricotómicos, ligeramente curvados hacia adentro, 0.3-0.5 mm de diámetro; células medulares de forma poliédrica con bordes romos, paredes anchas, 30-75 $\mu \mathrm{m}$ de diámetro, 2 capas de células corticales, poliédricas a irregulares; no se observaron estructuras reproductivas.

Hábitat: arena y rocas.

Ejemplares examinados: MÉXICO. Tabasco, municipio Paraíso, Puerto de dos Bocas, 26.V.2008, G. Hernández CA212 (UJAT).

Gracilaria venezuelensis W.R. Taylor, Allan Hancock Atlantic Exped. Rept. 2: 110. 1942. TIPO: VENEZUELA. Cubagua, Isla Margarita, $W . R$. Taylor-39-482 (holotipo: MICH). Fig. 3F.

Talo color púrpura-verdoso, aplanado en las zonas axilares y apicales; ramificaciones dicotómicas que se tornan más abundantes hacia los ápices; médula formada por 3 hileras de células poliédricas hialinas de paredes gruesas, células 65-120 $\mu \mathrm{m}$ de diámetro, 65-80 $\mu \mathrm{m}$ de largo; no se observaron estructuras reproductivas.

\section{Hábitat: rocas.}

Ejemplares examinados: MÉXICO. Tabasco, municipio Cárdenas, Puerto Andrés Sánchez Magallanes, 27.X.2012, L. Cabrera CA156 (UJAT); loc. cit., 27.X. 2012, J. Camarero CA173 (UJAT); loc. cit., 27.X. 2012,
A. Guillermo CA153 (UJAT); loc. cit., 27.X.2012, N. Quiroz et al. CA136 (UJAT).

\section{Rhodymeniales}

\section{Rhodymeniaceae}

Rhodymenia pseudopalmata (J.V. Lamouroux) P.C. Silva, Univ. Calif. Publ. Bot. 25: 265. 1952. TIPO: ESTADOS UNIDOS DE AMÉRICA. Sin localidad. Figs. 3G-I.

Talo comprimido, $5 \mathrm{~cm}$ de alto, 2-3 $\mathrm{mm}$ de ancho, color púrpura; márgenes con ondulaciones, bordes de los ápices romos y algunos ligeramente aguzados; ramificado dicotómicamente, en sección transversal 2 hileras de células medulares, poliédricas a irregulares, hialinas, 19$120 \mu \mathrm{m}$ de diámetro, 2 hileras de células subcorticales, 5-10.5 $\mu \mathrm{m}, 2$ capas de células de la corteza, fuertemente pigmentadas color rosa intenso, 2.5-5 $\mu \mathrm{m}$ diámetro; no se observaron estructuras reproductivas.

Hábitat: sobre sustrato artificial (plástico).

Ejemplares examinados: MÉXICO. Tabasco, municipio Cárdenas, Puerto Andrés Sánchez Magallanes, 27.X.2012, J. Arias CA3 (UJAT), CA179 (UJAT), GM71 (UJAT), GM394 (UJAT), GM400 (UJAT), GM403 (UJAT), GM406 (FCME).

\section{DISCUSIÓN}

Tomando en cuenta la información obtenida en el presente estudio y la revisión bibliográfica (Orozco-Vega y Dreckmann, 1995; Ramírez, 1996; Sentíes y Dreckmann, 2013) del grupo, en el estado de Tabasco, se han registrado 52 especies de rodofitas, ubicadas en tres clases, nueve ordenes, 17 familias y 32 géneros. De éstas, 21 especies se identificaron en el presente trabajo, de las cuales 10 fueron reportadas por Ramírez (1996) y más recientemente 10 por Sentíes y Dreckmann (2013) (Cuadro 2). Se presentan 12 nuevos registros de especies, que represen- 

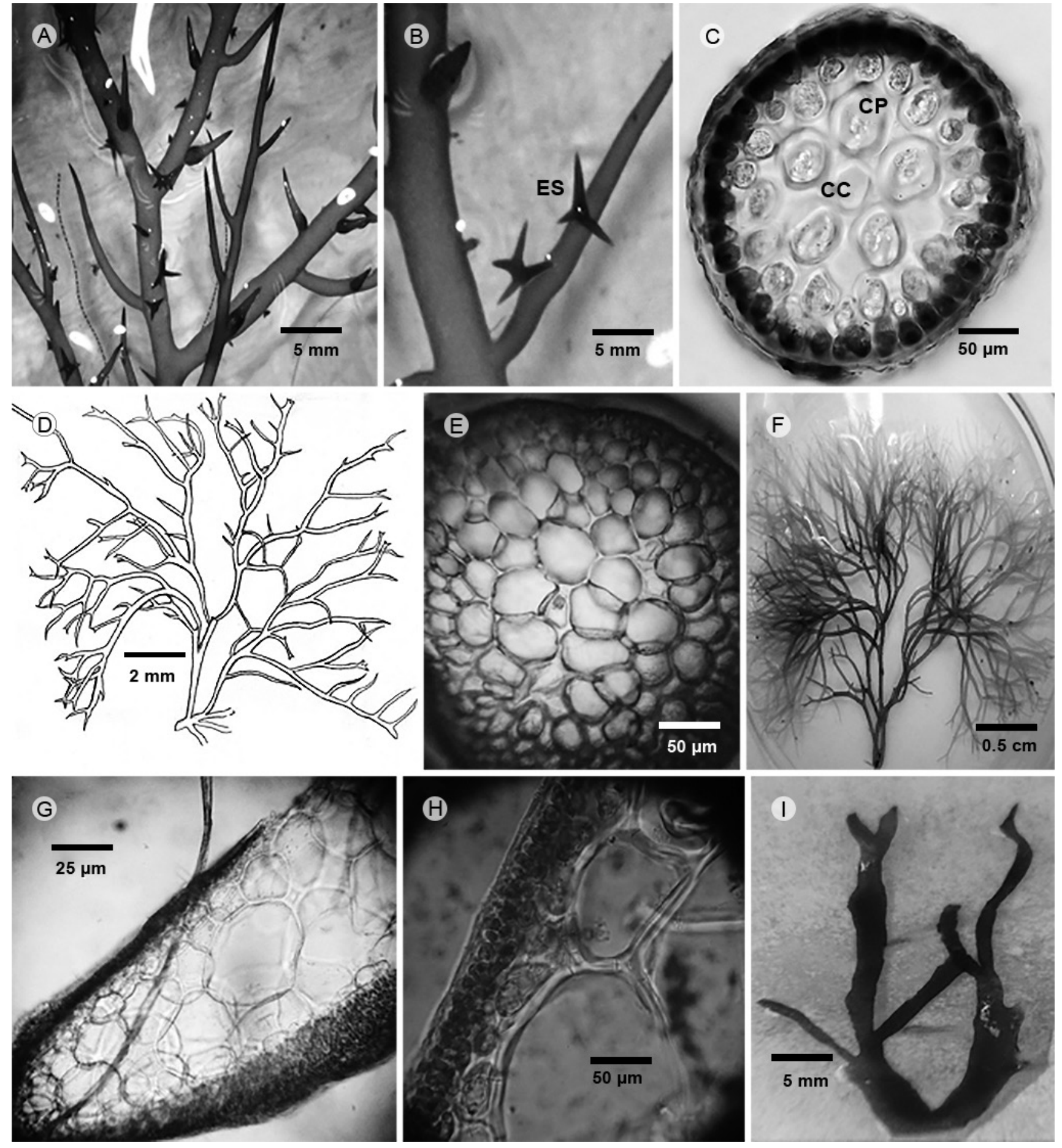

Figura 3: A-C. Hypnea valentiae (Turner) Montagne: A. aspecto del talo; B. detalle del talo con ramas con forma de estrella (ES); C. corte transversal, célula central (CC) y células pericentrales (CP). D, E. Gracilaria tikvahiae McLachlan: D. aspecto del talo; E. corte transversal. F. Gracilaria venezuelensis W.R. Taylor: aspecto del talo. G-I. Rhodymenia pseudopalmata (J.V. Lamouroux) P.C. Silva: G. corte transversal; H. detalle del corte transversal, se observan células medulares; I. aspecto del talo. 
$\tan 8 \%$ del número total de macroalgas marinas citadas para el Atlántico mexicano (Pedroche y Sentíes, 2003), así como $14 \%$ del número de especies para el Golfo de México (Ortega et al., 2001).

Según lo presentado por Ortega et al. (2001), Callejas-Jiménez et al. (2005), González-Gándara et al. (2007), Galicia-García et al. (2013), Mateo-Cid et al. (2013) y Sentíes y Dreckmann (2013), las rodofitas determinadas en el área de estudio presentan afinidad con las reportadas en Veracruz y Campeche. De las 52 especies de rodofitas registradas para Tabasco, hay una coincidencia para el litoral de Campeche de 36 especies, mientras que 39 también son similares para Veracruz, mostrando $70-76 \%$ de distribución continua entre las tres entidades federativas.

Como lo señalan Zavala et al. (2005) y QuirozGonzález et al. (2017), la afinidad que muestra Tabasco respecto a los estados colindantes puede explicarse por la presencia de corrientes costeras de la plataforma de Tamaulipas que circulan sobre Veracruz y Tabasco, hasta Campeche, especialmente en otoño e invierno. Estas corrientes tropicales asociadas a los intervalos de temperatura determinan qué especies son las que migrarán, y de acuerdo a las características físicas de muchas costas, definirán que especies se establecerán (Ramírez, 1996).

En comparación con el conocimiento que se tiene sobre la biodiversidad de algas rojas en el resto de los estados costeros del país, Tabasco aún presenta una riqueza específica baja de Rhodophyta, tomando en cuenta que el resto de las entidades, con excepción de Chiapas, presentan entre 70 y 452 especies en sus inventarios florísticos. Los registros en los estados del Golfo de México oscilan entre 87 y 230 especies (Pedroche y Sentíes, 2003) y presentan una diferencia notable respecto al número de especies registradas para Tabasco. Esto no implica una falta de distribución continua sino una falta de exploraciones ficológicas exhaustivas tal como se han llevado a cabo en el resto de los estados costeros del país.

La localidad con mayor número de especies fue el Puerto Andrés Sánchez Magallanes (Cárdenas) con 15, mientras que en el municipio Centla no se presentaron registros. Esta distribución de las especies está directamente relacionada con las características del área de estudio. La ausencia de algas en Centla podría deberse a la pobreza de sustratos sólidos, donde se fijan las algas en mayor proporción, ya que dicha localidad se define por playas limo-arcillosas y desembocaduras de los ríos González, Grijalva, así como del río San Pedro y San Pablo, mismos que modifican el aporte de nutrientes, sedimentos y salinidad de la costa (Quiroz-González et al., 2017).

Taylor (1960) señala que los sustratos expuestos al oleaje, como las escolleras, exhiben diversidad de algas marinas, tal como sucede en la Escollera del Puerto Andrés Sánchez Magallanes. Especies como Centroceras clavulatum, Acanthophora spicifera, Bryocladia cuspidata, Polysiphonia subtilissima, Gelidium pusillum, Hypnea valentiae, Gracilaria blodgettii y Grateloupia filicina toleran exposición al fuerte oleaje y a la desecación y son comunes en estos sitios. Este tipo de sustratos artificiales son una influencia de origen humano cada vez más común, mismos que comenzaron a citarse en estudios ficológicos a partir de 1958 (Garduño-Solórzano et al., 2005).

Las conchas de moluscos fueron los sustratos de origen animal más comunes, mismos que son considerados hospederos de numerosas comunidades algales dado que representan una excelente forma de dispersión. Seis especies (Centroceras clavulatum, Acanthophora spicifera, Bryocladia thyrsigera, Polisyphonia subtilissima, Gracilaria blodgettii y G. filicina) se encontraron sobre conchas, siendo las de ostión (Crassostrea virginica) las más comunes. Acanthophora spicifera se observó sobre algunos caracoles con vida que no fueron identificados taxonómicamente. Melanothamnus sphaerocarpus e Hypnea valentiae se observaron como epífitas de pastos marinos (Thalassia testudinum), la primera ha sido reportada por otros autores para el mismo sustrato (Mateo-Cid et al., 2013). Trece especies se presentaron como epífitas de algas y pastos marinos, de las cuales Bangia fuscopurpurea, Erythrotrichia carnea y Sahlingia subintegra fueron epífitas en especies de rodofitas como Acanthophora spicifera y Gracilaria blodgetti, y en clorofitas como Chaetomorpha antennina y Cladophora vagabunda. Grateloupia filicina e Hypnea valentiae se registraron sobre 
mayor número de sustratos (seis y cinco respectivamente). Esta baja especificidad al sustrato pudiera explicarse por lo mencionado por Darley (1987) quienes lo asocian con un ambiente eutrofizado, donde la gran cantidad de nutrientes enmascaran las interacciones químicas que generan la especificidad, disminuyéndola drásticamente y permitiendo que el alga se encuentre en gran variedad sustratos.

La ausencia de sustratos adecuados para el establecimiento de las algas en Tabasco es la principal razón para la poca riqueza especifica registrada hasta el momento, debido a la falta de sustratos rocosos, y a la prevalencia de sitios arenosos o con desembocaduras de ríos que modifican el aporte de nutrientes y la salinidad, situación que se suma a la falta de exploraciones ficológicas en la región.

Con base en Dawes (1991), la ficoflora del estado de Tabasco queda comprendida dentro del intervalo de distribución tropical de las algas marinas en relación con la temperatura del agua $\left(25^{\circ} \mathrm{C}\right)$, aspecto también señalado por Sentíes y Dreckmann (2013) quienes, basados en el índice de Cheney (1977), mencionan que la flora algal está cercana a iniciar una zona de transición hacia la flora caribeña. Destacan el valor biogeográfico de Tabasco, dada su condición de región aislada en términos de la dinámica geológica y oceanográfica, además de sus características de aguas someras y calmadas.

Gracilaria pacifica únicamente ha sido registrada por Orozco-Vega y Dreckmann en 1995 para la laguna de Mecoacán. Si bien en el presente trabajo se incluye en el listado taxonómico, es importante llevar a cabo una evaluación de si se trata realmente de esta especie o más bien de una determinación errónea debido a que ha sido registrada para las costas del Pacífico mexicano.

De las 52 especies de rodofitas listadas para el estado de Tabasco 10 han sido señaladas con algún tipo de uso medicinal, alimenticio o industrial, lo que incrementa el interés en su estudio (Quiroz-González y Rivas-Acuña, 2014).

\section{CONCLUSIONES}

Se considera importante continuar realizando exploraciones ficológicas a lo largo de la costa del estado de Tabas- co, principalmente en el municipio Centla y en las lagunas costeras como Laguna del Carmen y Mecoacán, con el fin de profundizar el conocimiento de la ficoflora de la región en la zona intermareal e inclusive sublitoral. Información adicional sobre la distribución, la estacionalidad, la morfología, la anatomía y la reproducción de las algas son importantes de abordar, ya que repercuten en nuevas investigaciones útiles en la solución de problemas taxonómicos y el aprovechamiento sustentable de recursos algales.

\section{CONTRIBUCIÓN DE AUTORES}

NQ, DL y MR llevaron a cabo las recolectas de material en las áreas de muestreo. NQ realizó la revisión del material de herbario, la elaboración de preparaciones, la toma de medidas, y la identificación y descripción de los ejemplares. DL y MR corroboraron la identificación taxonómica. NQ escribió el manuscrito con el apoyo de DL y MR. Todos los autores contribuyeron a la discusión, revisión y aprobación del manuscrito final.

\section{FINANCIAMIENTO}

El estudio no tuvo financiamiento por parte de ningún proyecto.

\section{AgradeCIMIENTOS}

Agradecemos a Silvia Capello García y María de los Ángeles Guadarrama, por el apoyo brindado con los equipos e instalaciones para el procesamiento e identificación de las muestras a Miguel A. Ortiz Ramos por su apoyo en campo y a Luis Gabriel Aguilar Estrada por su apoyo en la elaboración del mapa.

\section{LITERATURA CITADA}

Ardito, S. y M. García. 2009. Estudio ficológico de las localidades de Puerto Francés y San Francisquito, Estado Miranda, Venezuela. Acta Botanica Venezuelica 32(1): 113-143.

Callejas-Jiménez, M. E., A. Sentíes y K. M. Dreckmann. 2005. Macroalgas de Puerto Real, Faro Santa Rosalía y Playa Preciosa, Campeche, México, con algunas consideraciones florísticas y ecológicas para el estado. Hidrobiológica 15(1): 89-96. 
Castellaro, G., F. Squella, T. Ullrich, F. León y A. Raggi. 2007. Algunas técnicas microhistológicas utilizadas en la determinación de la composición botánica de dietas de herbívoros. Agricultura Técnica 67(1): 86-93. DOI: https://doi.org/10.4067/s0365-28072007000100011

Castro-Nunes, J. 2007. Duas espécies de Rhodymenia (Rhodophyta, Rhodymeniaceae) no Estado da Bahia, Brasil. Acta Botanica Malacitana 32: 228-232.

Cheney, D. P. 1977. R \& C/P A new and improved ratio for comparing seaweed floras. Journal of Phycology 13(supp. 2): 3-78. DOI: https://doi.org/10.1111/j.1529-8817.1977. tb02421.x

Darley, M. 1987. Biología de las Algas, enfoque fisiológico. Ed. Limusa. México, D.F., México. 518 pp.

Dawes, C. 1991. Botánica marina. Ed. Limusa. México, D.F., México. 673 pp.

De la Garza, A. 2003. Clave genérica de las algas rojas marinas macroscópicas y comunes de las costas de Veracruz, México. Tesis de licenciatura. Facultad de Ciencias, Universidad Nacional Autónoma de México. México, D.F., México. 74 pp.

Díaz-Tapia, P., L. McIvor, D. W. Freshwater, H. Verbruggen, M. J. Wynney y C. A. Maggs. 2017. The genera Melanothamnus Bornet \& Falkenberg and Vertebrata S.F. Gray constitute well-defined clades of the red algal tribe Polysiphonieae (Rhodomelaceae, Ceramiales). European Journal of Phycology 52(1): 1-30. DOI: https://doi.org/10 $.1080 / 09670262.2016 .1256436$

Dreckmann, K. y G. De Lara-Isassi. 2000. Gracilaria caudata J. Agardh (Gracilariaceae, Rhodophyta) en el Atlántico mexicano. Hidrobiológica 10(2): 125-130.

Fernández, C. 2008. Flora marina del Parque Nacional Isla del Coco, Costa Rica, Pacífico Tropical Oriental. Revista de Biología Tropical 56(suppl. 2): 57-69.

Galicia-García, C., N. Robinson y Y. Okolodkov. 2013. New records of red algae (Rhodophyta) for Cabezo reef, national park sistema arrecifal veracruzano, Gulf of Mexico. Acta Botanica Mexicana 102: 39-76. DOI: https://doi.org/10.21829/abm102.2013.233

Garduño-Solórzano, G., J. Godínez-Ortega y M. M. Ortega. 2005. Distribución geográfica y afinidad por el sustrato de las algas verdes de las costas del Golfo de México y Mar Caribe. Boletín de la Sociedad Botánica de México 76: 61-78.

González-Gándara, C., M. V. Cruz-Arellano, C. DomínguezBarradas, A. Serrano-Solís y A. Basáñez-Muñoz. 2007. Macroalgas asociadas a cuatro hábitats del arrecife Tuxpan, Veracruz, México. Revista UDO Agrícola 7(1): 252-257.

González-González, J., M. Gold-Morgan, H. León-Tejeda, C. Candelaria, D. León-Álvarez, E. S. Zaragoza y D. Fragoso. 1996. Catálogo onomástico (nomenclator) y bibliografía indexada de las algas bentónicas marinas de México. Cuadernos No. 34. Instituto de Biología, Universidad Nacional Autónoma de México. México, D.F., México. 492 pp.

Guimarães, S., M. Fujii, D. Pupo y N. S. Yokoya. 2004. Reavaliação das características morfológicas e suas implicações taxonômicas no gênero Polysiphonia sensu lato (Ceramiales, Rhodophyta) do litoral dos Estados de São Paulo e Espírito Santo, Brasil. Revista Brasileira de Botânica 27(1): 163-183.

Guiry, M. y G. Guiry. 2017. AlgaeBase. World-wide electronic publication. National University of Ireland. Galway, Irlanda. http://www.algaebase.org (consultado octubre de 2016).

Littler, D. S. y M. M. Littler. 2000. Caribbean reef plants: an identification guide to the reef plants of the Caribbean, Bahamas, Florida and Gulf of Mexico. Offshore Graphics. Washington, D.C., USA. 542 pp.

Littler, D., M. Littler, K. Bucher y J. Norris. 1989. Marine plants of the Caribbean, a field guide from Florida to Brazil. Smithsonian Institution Press. Washington, D.C., USA. 263 pp.

Lot, A. y F. Chiang. 1986. Manual de herbario. Consejo Nacional de la Flora de México, A.C. México, D.F., México. 142 pp.

Mateo-Cid, L., C. Mendoza-González, A. Ávila-Ortiz y S. Díaz-Martínez. 2013. Algas marinas bentónicas del litoral de Campeche, México. Acta Botanica Mexicana 104: 53-92. DOI: https://doi.org/10.21829/ abm104.2013.57 
Mendoza-González, C. y L. Mateo-Cid. 1996. Contribución al estudio de la ficoflora marina de la costa del estado de Chiapas, México. Polibotánica 2: 61-118.

Mitsunobu, K., S. Lindstrom, T. Nakayama, A. Yokoyama, S. Mei Lin, M. Guiry, C. Gurgel, J. Huisman, T. Kitayama, M. Suzuki, T. Cho y W. Fre. 2017. Syllabus of Plant Families-A. Engler's Syllabus der Pflanzenfamilien Part 2/2: Photoautotrophic eukaryotic Algae-Rhodophyta. $171 \mathrm{pp}$.

Morón, M. y S. Ardito. 2010. Estudio ficológico del litoral rocoso de Isla Larga, Parque Nacional San Esteban, estado Carabobo, Venezuela. Acta Botanica Venezuelica 33(2): 167-185.

Orozco-Vega, H. y K. Dreckmann. 1995. Macroalgas estuarinas del litoral del Golfo de México. Cryptogamie, Algologie 16(3): 189-198.

Ortega, M. M., J. L. Godínez y G. Garduño Solórzano. 2001. Catálogo de algas bénticas de las costas mexicanas del Golfo de México y Mar Caribe. Comisión Nacional para el Estudio de la Biodiversidad-Universidad-Nacional Autónoma de México. México, D.F., México. 594 pp.

Pedroche, F. y A. Sentíes. 2003. Ficología marina mexicana. Diversidad y problemática actual. Hidrobiológica 13(1): 23-32.

Peruzzi, V., D. Rezende, N. Marcello, Y. Yoneshigue y R. Clapis. 2009. Four new additions to the marine flora of Fernando de Noronha Archipelago, Tropical western South Atlantic Ocean. CheckList 5(2): 210-215.

Quiroz-González, N. y M. Rivas-Acuña. 2014. Usos potenciales de las algas de Tabasco. Biodiversitas 115: 7-11.

Quiroz-González, N., D. León-Álvarez y M. G. RivasAcuña. 2017. Nuevos registros de algas verdes marinas (Ulvophyceae) para Tabasco, México. Acta Botanica Mexicana 118: 121-138. DOI: https://doi.org/10.21829/ abm118.2017.1204.

Ramírez, A. 1996. Estudio preliminar de las algas rojas (Rhodophyta) del litoral del Estado de Tabasco. Tesis de licenciatura. Universidad Nacional Autónoma de México, Campus Iztacala. Tlalnepantla, Estado de México, México. 66 pp.

Sánchez, A. y E. Barba. 2005. Capítulo I: Biodiversidad de Tabasco. In: Bueno, J., F. Álvarez y S. Santiago (eds.). Biodiversidad del Estado de Tabasco. Instituto de Biología, Universidad Nacional Autónoma de MéxicoComisión Nacional para el Conocimiento y Uso de la Biodiversidad. México, D.F., México. pp. 1-16.

Sentíes, A. y K. Dreckmann. 2013. Lista actualizada de las macroalgas de Tabasco, México. Acta Botanica Venezuelica 36(2): 109-117.

Solé, M. y P. Pardo. 2010. Ficoflora Marina del Sur de la Península de Macanao, Isla de Margarita, Venezuela. II. Rhodophyta. Acta Botanica Venezuelica 33(2): 187-211.

Solé, M. y A. Suárez. 2010. Adiciones a las Rhodophytas de Cuba. Revista Investigaciones Marinas 31(1): 3-9.

Taylor, W. 1960. Marine algae of the Eastern Tropical and Subtropical Coasts of the Americas. University of Michigan Press. Michigan, USA. 823 pp.

West, R., N. Psuty y B. Thom. 1985. Las tierras bajas de Tabasco en el Sureste de México. Instituto de Cultura de Tabasco. Villahermosa, México. 416 pp.

Won, Y., S. Fredericq y T. Cho. 2011. Two new species of Centroceras (Ceramiaceae, Rhodophyta) from KwaZulu-Natal, South Africa. European Journal of Phycology 45(3): 240-246. DOI: https://doi. org/10.1080/09670261003746219

Zavala, J., O. Salmeron, V. Aguilar, S. Cerdeira y M. Kolb. 2005. Caracterización y regionalización de los procesos oceanográficos de los mares mexicanos. Comisión Nacional para el Conocimiento y Uso de la Biodiversidad. México, D.F., México. http://www.conabio.gob.mx/gap/ index.php/Procesos_oceanogr\%C3\%A1ficos. (consultado marzo de 2017). 